\title{
Instrumentation of Data Acquisition for EIT
}

\author{
Sukanya Aldar \\ Electronics \\ Department \\ S.P.I.T., Mumbai, \\ India
}

\author{
Shruti Chavan \\ Electronics \\ Department \\ S.P.I.T., Mumbai, \\ India
}

\author{
Dakshata Patil \\ Electronics \\ Department \\ S.P.I.T., Mumbai, \\ India
}

\author{
Priya Chimurkar \\ Electronics \\ Department \\ S.P.I.T., Mumbai, \\ India
}

\begin{abstract}
Electrical Impedance Tomography is a medical imaging technique which maps the resistivity distribution of the body and find the location of abnormalities present in the body part. In present techniques of imaging like CT scan, MRI and Ultrasound the patients have to move in specialized Room and bedside monitoring using these machines are not possible while it is possible by using EIT even in Intensive Care Unit where patients cannot be moved. This paper focuses on Instrumentation for Data acquisition for detection of location of cancerous tumors using Electrical Impedance Tomography. As we cannot experiment directly on the body, we are experimenting on phantom using saline water with adjacent current injection protocol having 16 electrodes attached on the periphery of Phantom. Our proposed research will be low cost, high speed and accurate solution for Data acquisition used in Imaging of EIT. This paper discussed on design of constant current source and Instrumentation for fast and accurate acquisition of voltages. Total 208 readings of voltages are taken by using switching circuit which is developed using Multiplexer, Demultiplexer, myRIO student embedded device and LabView.
\end{abstract}

\section{General Terms}

Electrical impedance tomography, LabView based advance instrumentation,

\section{Keywords}

Analog multiplexer, Electrical impedance tomography, LabView, myRIO, Instrumentation

\section{INTRODUCTION}

Electrical Impedance Tomography (EIT) is an imaging technique from two decades in which distribution of conductivity is calculated by placing electrodes on the surface of the body [1]. The objective of the EIT is to obtain the spatial distribution of the conductivity inside the human body and interpret it as an image [2].

Most commonly used methods for detection and monitoring of cancer are CT Scan, X-rays, MRI and Ultrasound. These techniques have impediments like harmful radiation which may cause side effects to the body [2]. Also, these machines are costly and not portable. Specialized room is required for diagnosis using these machines.

These all, limitations are taken care in EIT. In EIT, average differential voltage measurements with respect to adjacent current injection approach are used to form a tomographic image of tissues of human body. EIT does not lead to harmful radiation. Also, the magnitude of alternating current which is used as an input in this technique is very less (in $\mathrm{mA}$ ) [3] due to which it won't cause any nerve stimulation. The operating frequency for alternating current is moderately high $(10 \mathrm{KHz}$ to $50 \mathrm{KHz}$ ) so that it won't give rise to electrolytic effect in the human body [4]. Also, the ohmic power dissipation is less and it is diffused over the body which can be manageable by human thermo-regulatory system which makes EIT technique suitable for human body.

Due to the advantages of EIT like non-invasiveness, portability and radiation free, EIT is being studied and researched since 1970.Presently EIT is accepted as Gold method for the application in pulmonary monitoring, regional ventilation inside lungs and lung volume monitoring in hospitals. In this paper we are proposing EIT as a method for detection of type and location of Cancerous tumor in body [4]. There are two types of tumor, benign tumor which is analogous to non-conducting impurity while malignant tumor is analogous to conducting impurity [3].

Fig...01 is the block diagram of EIT system. Constant current is injected to the electrode pair and voltage from other pair of electrodes is acquired using switching circuit. Phantom having

16 electrodes attached to it is connected to switching circuit. For carrying switching operation and voltage acquisition between the electrodes myRIO and LabView is used.

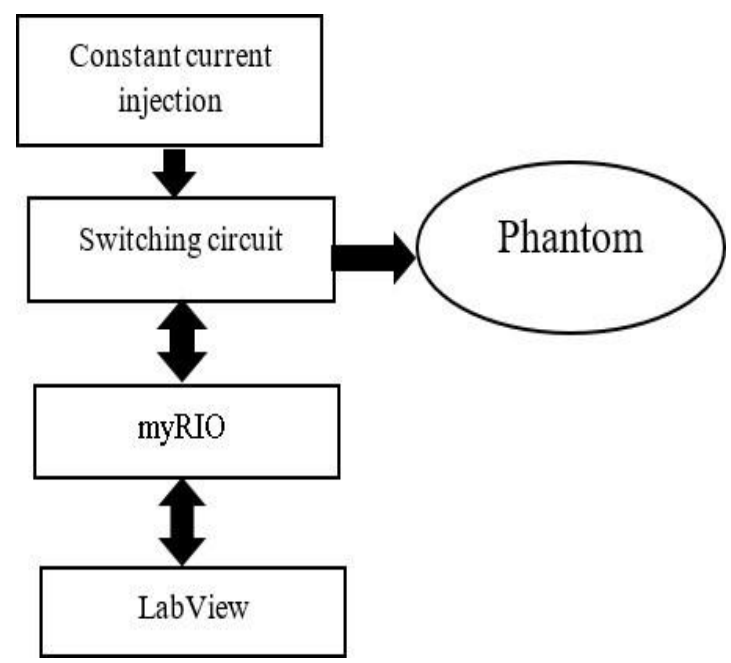

Fig.1 Block Diagram of EIT system

\section{EIT SYSTEM}

A circular plastic box of $15 \mathrm{~cm}$ diameter has been used to make a closed phantom. The ECG electrodes are placed around the circumference of box equidistant from each other at an angle of 22.5 We have developed Automatic Data Acquisition system for Electrical Impedance Tomography using LabVIEW software, in which adjacent current injection approach is used for measurement. Fig.02 shows switching circuit of this system. 16 electrodes are placed at the surface of circular phantom. Alternating current as an input to a 
phantom is provided through a constant current source using LM334. CD4067 is a single 16 channel analog multiplexer and demultiplexer IC. A 16 electrodes EIT system requires two 16:1 analog multiplexers (CD4067) and two 1:16 analog demultiplexers (CD4067) in order to establish a switching network [5]. Automatic voltage data acquisition is done using LabView 2015. Select lines of CD4067 are connected to digital I/O pins of myRIO. Since myRIO has 16 Digital I/O pins single myRIO is enough for carrying switching operation for 16 electrodes.

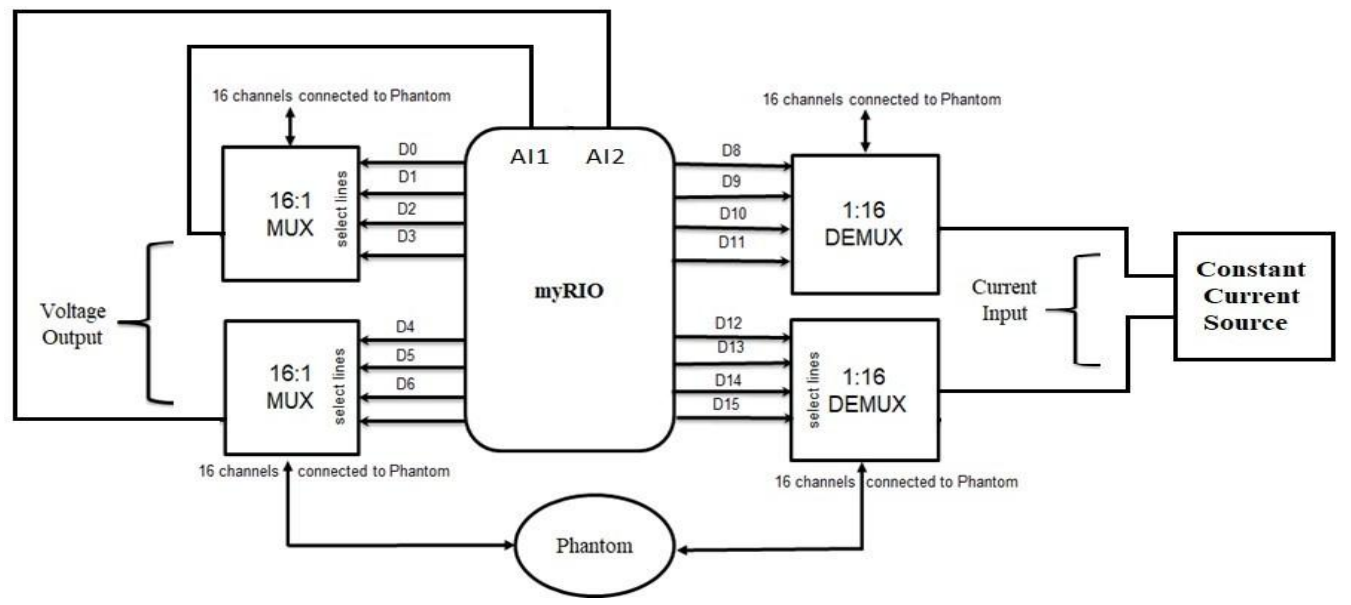

Fig.2 Switching circuit

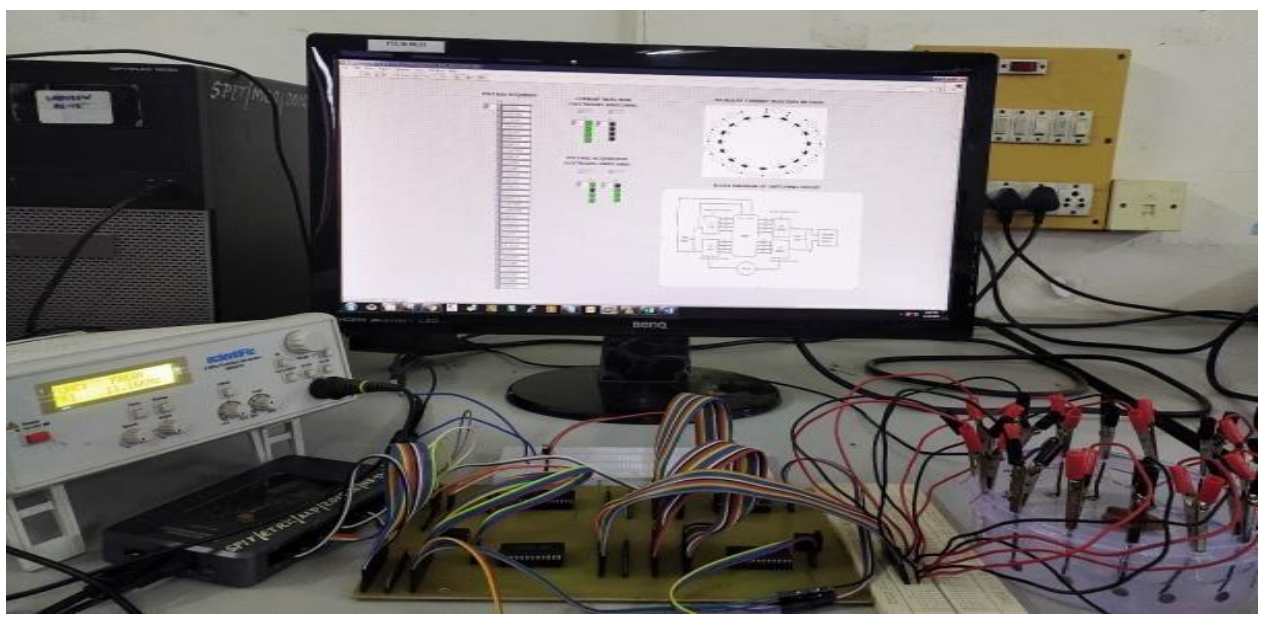

Fig.3 Hardware setup

Impedance and Diffused Optical Reconstruction Software) which is an open source software used for the reconstruction ofEIT. For acquiring voltage from Analog pin of myRIO is used which is connected to output of two multiplexer ICs. myRIO is a real time embedded board for evaluation which can be programmed by using LabVIEW. myRIO is an efficient device for performing automatic switching operation of electrodes and measuring differential voltages. Its processing speed is almost ten times that of the standard general microcontrollers and microprocessor [7]. As soon as the switching network completes one cycle of measuring differential voltages with respect to current injection then it will get switched to adjacent electrodes and the same procedure will be done till all the adjacent pairs of electrodes are done. myRIO is used for data acquisition i.e. instantaneous differential voltage measurement from the electrodes. All the differential voltages are stored in the database and by using the results, graph has been plotted in LabVIEW software as shown in Fig.4. This differential voltage is then used for image reconstruction in EIDORS to get approximate size of impurity present. EIDORS (Electrical. 


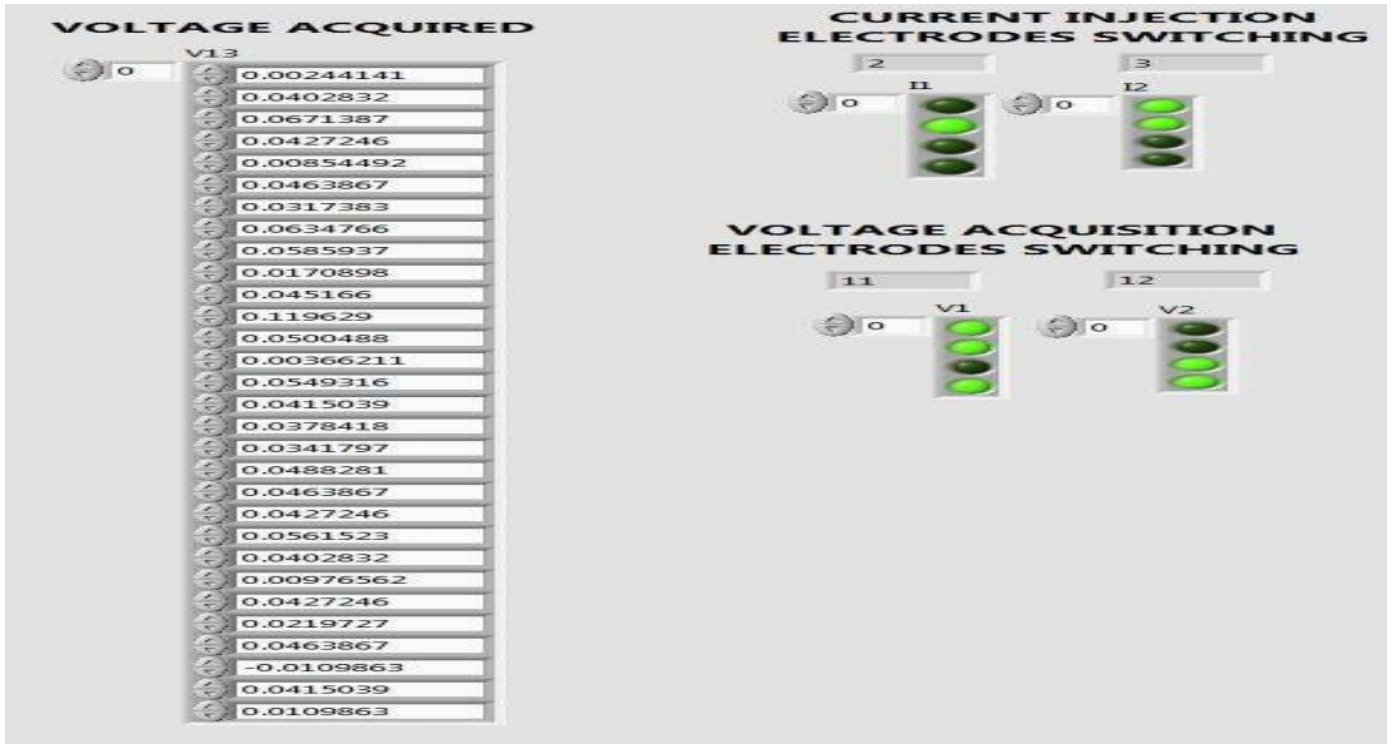

Fig.4 LabView based graphical user interface

Fig.5 shows the simulation of LM334 which provides constant current of $1 \mathrm{~mA}$ [8]. An external Resistor R1 is connected which will give constant current. Input $\mathrm{AC}$ voltage is given of $5 \mathrm{~V}, 10 \mathrm{KHz}$.

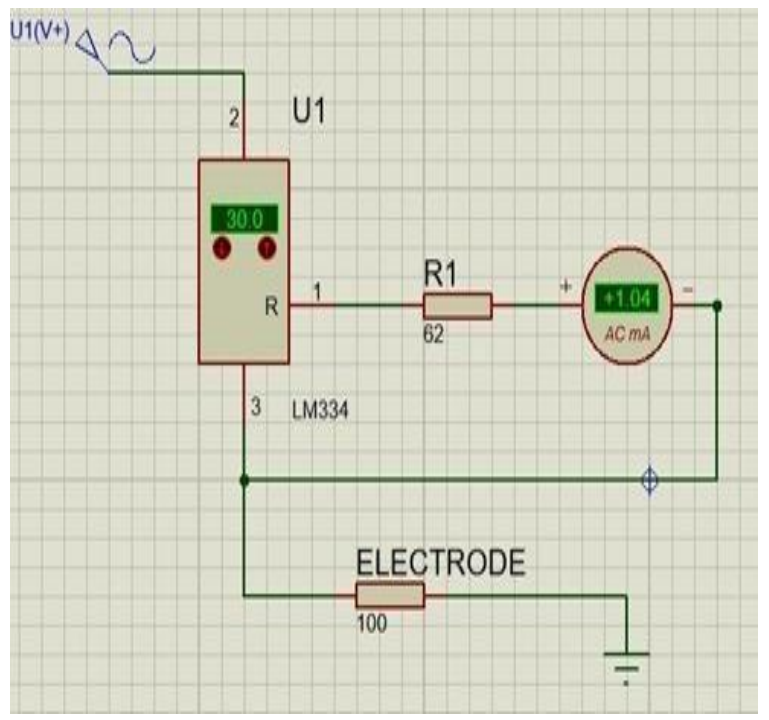

Fig.5 Constant current source

\section{EIT INSTRUMENTATION}

\subsection{Normal or Body Text}

Fig.06 and Fig.07 are showing adjacent current injection method which is used here. In this method first current is given between electrode 1-2 and voltage is taken between the pairs of electrodes 3-4, 4-5, 5-6,6-7 till 15-16. In the next cycle current is given between electrode pair 2-3 and voltage is taken between other pairs of electrodes 4-5, 5-6,6-7 till 161. In the same way procedure gets repeated for all the electrodes pair. After getting this procedure repeated for all the other pairs total voltage readings, we will get will be 208 . Taking these 208 readings manually will require large time so we have developed automatic data acquisition system using myRIO and LabVIEW which will take readings automatically [2]. A myRIO is a real time embedded evaluation board which can be programmed using LabVIEW. Its processing speed is almost ten times that of the standard general microcontrollers and microprocessor.

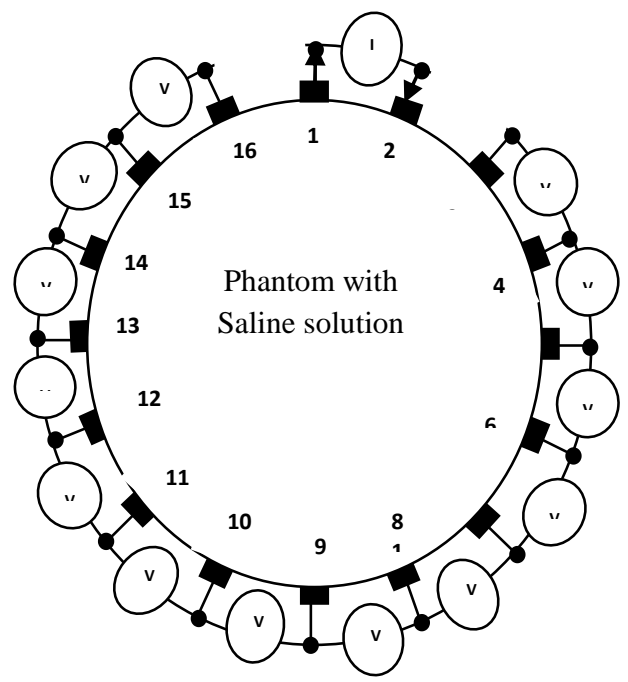

Fig.6 Projection 1 [9]

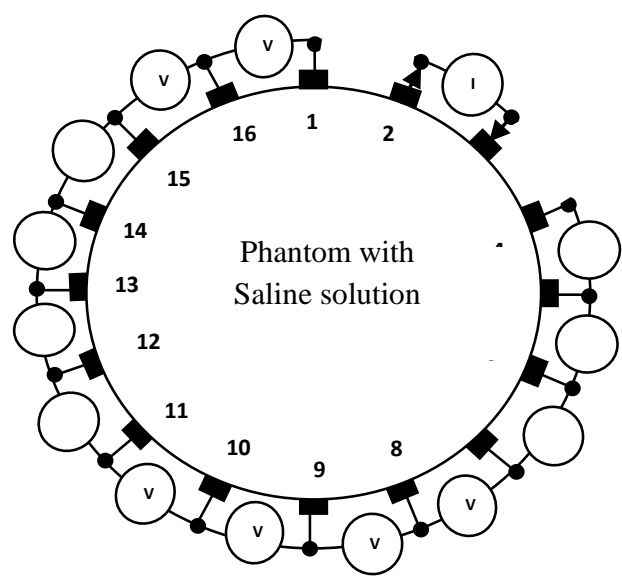

Fig.7 Projection 2 [9] 
Fig.08. shows switching network for carrying out the switching operation which consists of analog multiplexer and demultiplexer CD4067 whose 16 channels are connected to electrodes attached to phantom and select lines are controlled using myRIO. 5V supply is given using adapter to this circuit. Output of multiplexer is used for acquiring voltages from electrodes which are connected to analog input pin of myRIO and input to demultiplexer is the constant current source which is given externally using constant current source circuit developed using LM334.

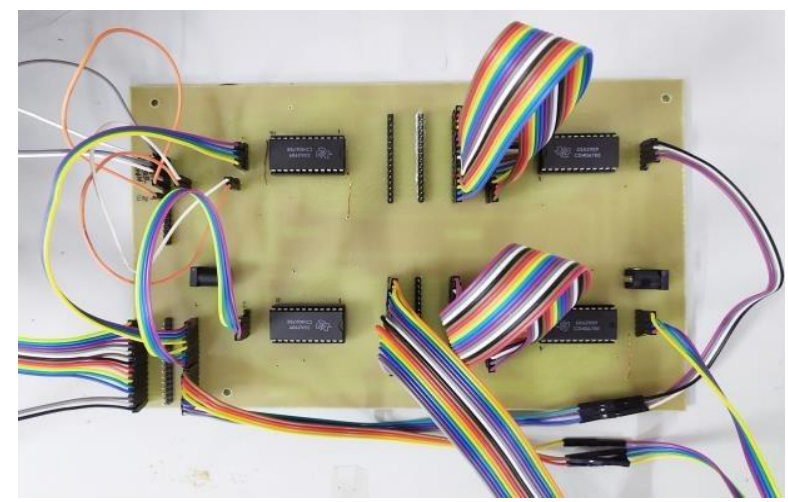

Fig.8 Switching network

\section{FLOWCHART}

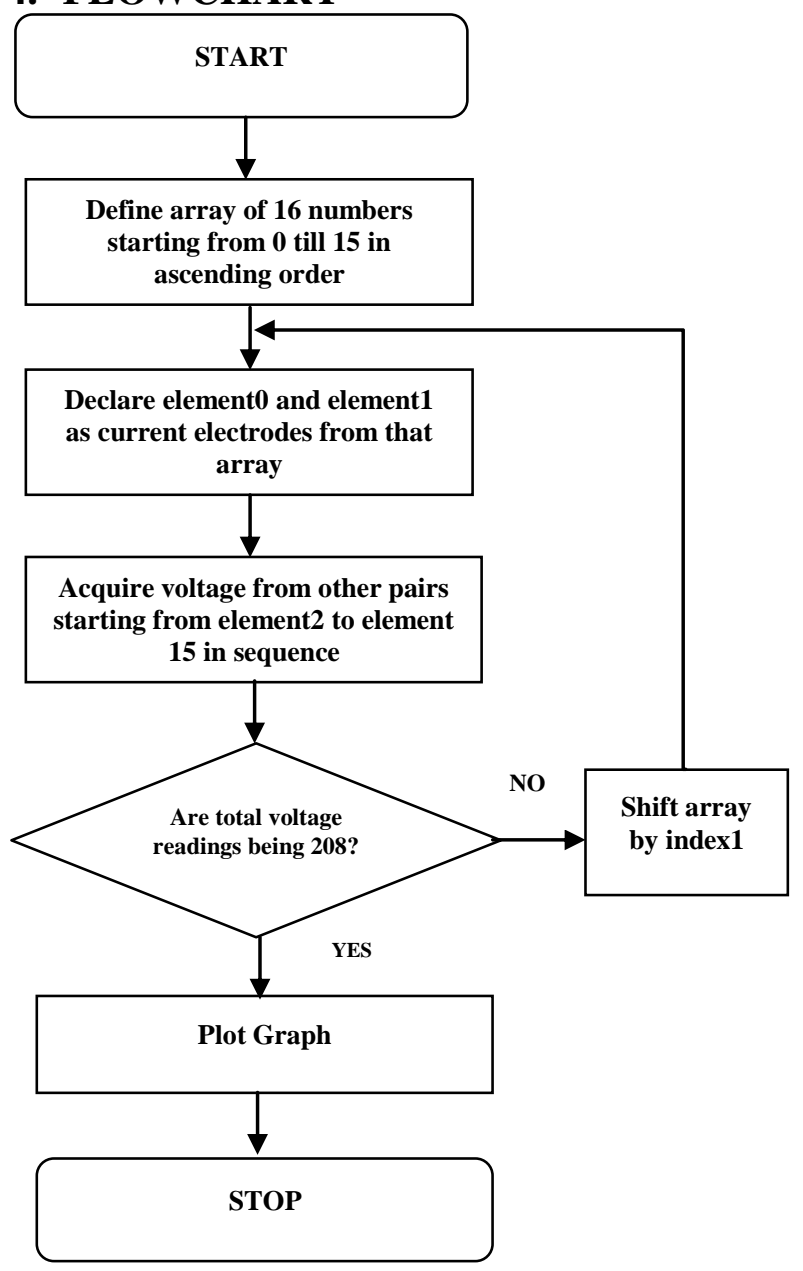

Fig.9 Simulation Flowchart in LabVIEW

Fig.09 shows for carrying switching operation array of 0 to 15 numbers have been declared in the program where element at index 0 and index 1 will always be current electrodes. Element at index 2 to index 15 are taken in pairs in sequence for voltage acquisition. After acquiring voltage from 13 pairs, arrayelements are shifted by 1 . Again, procedures repeat until total voltage readings are not equal to $208\left(16^{*} 13\right)$. These readings are plotted on graph.

\section{SIMULATION RESULT}

In Fig.10 graph is plotted by taking readings between the electrode pairs with and without impurity. Graph in orange colour is for readings which was taken without impurity, Graph in blue colour is showing readings taken when impurity was present. Non-Conducting impurity stone was kept near electrode_16. In the graph we can observe the change in reading near electrode_16.

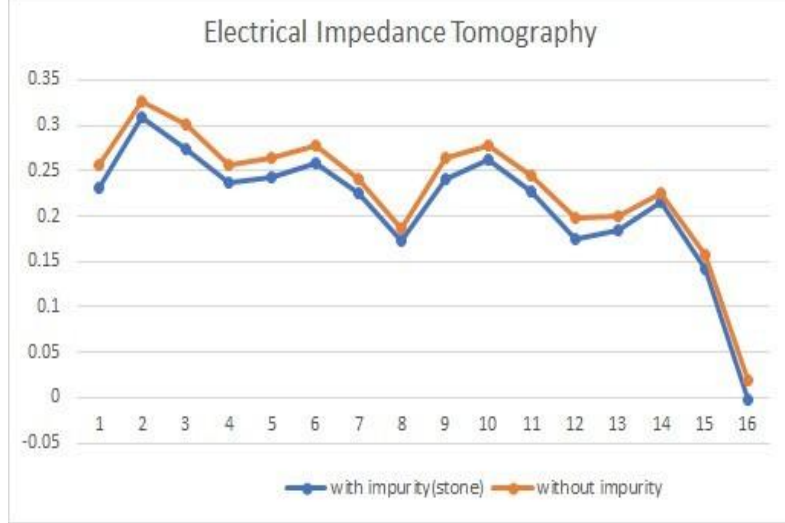

Fig.10. Voltage acquired result

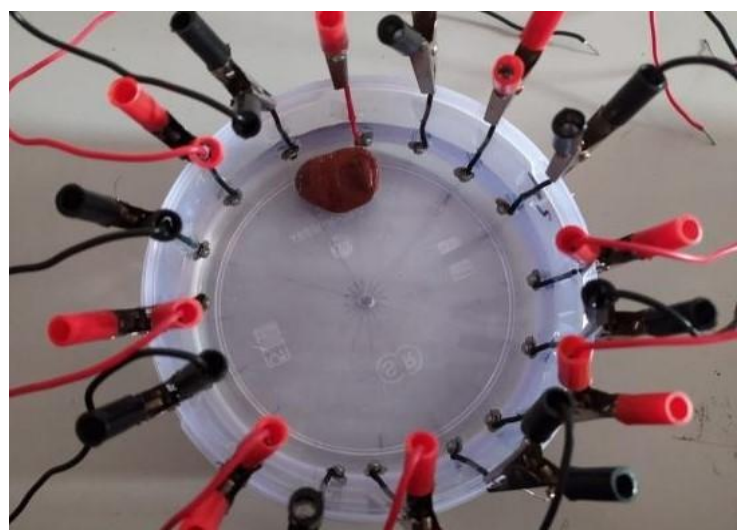

Fig.11. Phantom with stone impurity

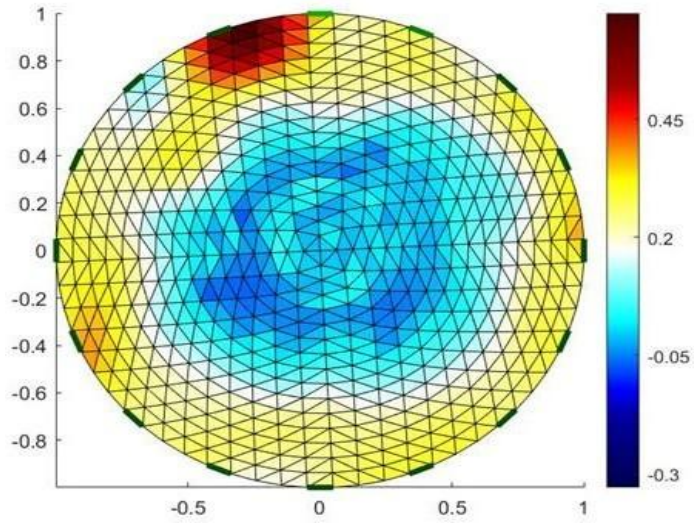

Fig.12. Image reconstruction result

Fig.11 and Fig.13 shows the phantom image when impurity stone was added in saline solution. Using EIDORS (Electrical 
Impedance and Diffused Optical Reconstruction Software) reconstruction of EIT is done. The non- conducting impurity used for experiments is stone. The database of differential

voltages is loaded in MATLAB software. Gauss Newton inverse algorithm is applied for getting reconstructed image showing the distribution of the conductivity inside the human body. This data is given to EIDORS software for image reconstruction which will show the impurity present at exact position[7][8]. Fig.12 and Fig.14 shows image reconstructed when impurity was added.

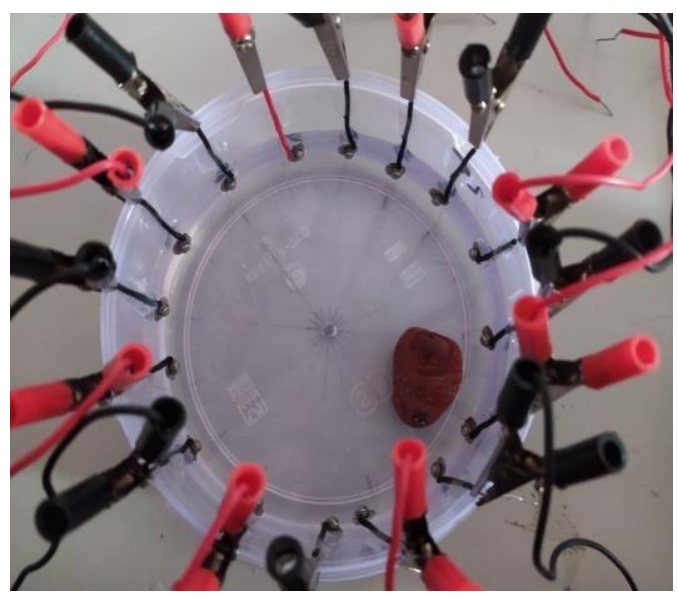

Fig.13. Phantom with stone impurity

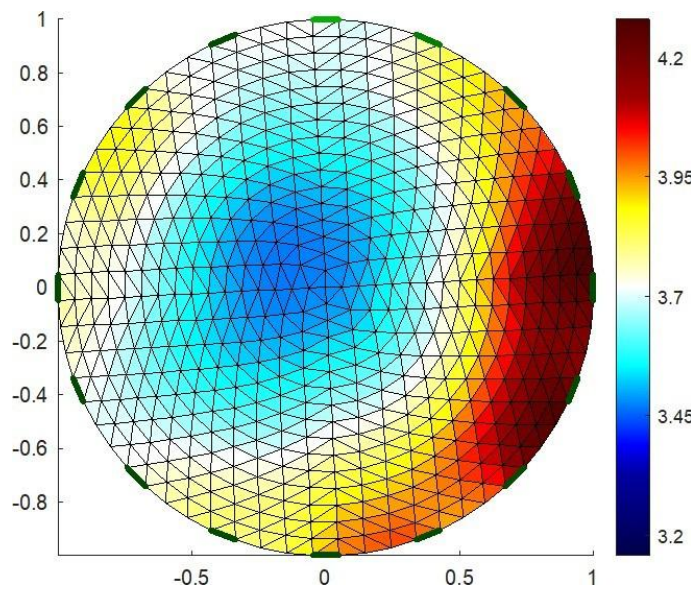

Fig.14. Image reconstruction result

\section{CONCLUSION}

In this study, automatic data acquisition system is designed using myRIO for Electrical Impedance Tomography. Adjacent current injection techniques for input stage i.e. constant current source at $1 \mathrm{~mA}$ current and $10 \mathrm{KHz}$ frequency is used which gives better response. Also, myRIO is useful for acquiring the data at a faster rate. From the entire resistivity imaging studies using Electrical Impedance Tomography, it is concluded that distribution of impedance in images are successfully acquired from the boundary data collected from the impurity tissues from phantom with adjacent current injection method. Thus, position of cancerous tissue can be detected. Instrumentation for Data acquisition for detection of location of cancerous tumors using Electrical Impedance Tomography is developed. When non conducting impurity is kept i.e. stone in saline solution low conductivity distribution is observed where impurity is placed which shows presence of benign tumour. Thus, the type of cancer could be recognized non-invasively and with no radiation.

\section{REFERENCES}

[1] Tushar Kanti Bera, Student Member IEEE and J. Nagaraju: "Switching of The Surface Electrode Array in A 16-Electrode EIT System Using 8-Bit Parallel Digital Data”, 2011 World Congress on Information and Communication Technologies

[2] Vidya Sarode, Hema Patil, Alice N. Cheeran: "Labview Based Automatic Data Acquisition system for Electrical Impedance Tomography", International Journal of Engineering Science and Technology 2014 Vol(5)

[3] J. G. Webster, Electrical impedance tomography, Adam Hilger series1221 of biomedical engineering, Adam Hilger, New York (USA), 1990.

[4] Tushar Kanti Bera: "Applications of Electrical Impedance Tomography (EIT): A Short Review", doi:10.1088/1757-899X/331/1/012004, 2018 IOP Conf. Ser.: Mater. Sci. Eng. 331012004

[5] Datasheet Texas instrument CD4067: https://www.ti.com/lit/ds/symlink/cd406 7 b.pdf, June 2003

[6] Gang Ye, Kim H. Lim, Rhett George, Gary Ybarra, William T. Joines \& Qing H. Liu, “ A 3D EIT System for Breast Cancer Imaging", 3rd IEEE Symposium on Biomedical Imaging, pp. 1092- 1095, 2006.

[7] DatasheettexasinstrumentLM334:http://www.ti.com/lit/d s/symlink/lm134.pd f , May 2013

[8] Nick Polydorides and William R B Lionheart: "A Matlab toolkit for three-dimensional electrical impedance tomography: a contribution to the Electrical Impedance and Diffuse Optical Reconstruction Software project", PII: S0957- 0233(02)35899-5, Meas. Sci. Technol. 13 (2002) 1871-1883

[9] Vidya Sarode, Priya M. Chimurkar and Alice N. Cheeran: "Electrical Impedance Tomography using EIDORS in a Closed Phantom", DOI: 10.5120/74600526 Volume 48- No.19, June 2012

[10] Vidya Sarode, Sneha Patkar and Alice N. Cheeran, "Comparison of 2-D Algorithms in ElT based Image Reconstruction", International Journal of Computer Applications (0975 - 8887) Volume 69-No.8, May 2013

[11] User guide and specification NImyRIO1900:http://www.ni.com/pdf/manuals/376047c.pdf, May2016 